\title{
Factors Affecting Development of Alternaria Leaf Spot of Asalio Caused By Alternaria Alternata And Identification of Pathogen
}

Poonam Kumari ( $\sim$ poonamkumarippathjin@gmail.com )

Rajasthan College of Agriculture

Amit Trivedi

Rajasthan College of Agriculture

Akansha Deora

Rajasthan College of Agriculture

Neelam Geat

Department of Plant Pathology

Lokesh Kumar

Rajasthan College of Agriculture

\section{Research Article}

Keywords: Alternaria alternata, host age, inoculum density, internal transcribed spacer (ITS) regions, Asalio, Alternaria leaf spot disease.

Posted Date: December 1st, 2021

DOI: https://doi.org/10.21203/rs.3.rs-1051824/v1

License: (c) (1) This work is licensed under a Creative Commons Attribution 4.0 International License. Read Full License 


\section{Abstract}

The present study was on management of Alternaria leaf spot caused by Alternaria alternata in Asalio. The disease was found to be very severe in all Asalio growing areas causing heavy destruction. The main aim of this investigation was to find out effect of age of the host, inoculum density in Alternaria leaf spot disease development and molecular identification of pathogen. The result shows that the susceptibility in Asalio increased with increase in age on inoculatios of $A$. alternata. Maximum disease was obtained in the plants ageing 40 DAS with PDI 55.60 per cent. Among the variously examined levels of inoculum concentrations. The two lower concentrations $1 \times 10^{1}$ and $1 \times 10^{2}$ conidia ml$^{-1}$ exhibited significant difference in the PDI (20.00 and 31.20 respectively) within each other. $1 \times 10^{3}$ and $1 \times 10^{4}$ conidia ml $^{-1}$ inoculum levels were found to be significantly at par to each other with PDI (39.20 and 40.80 respectively). $1 \times 10^{3}$ conidia $\mathrm{ml}^{-1}$ inoculum concentration was optimum for causing highest level of disease. After inoculation of inoculum $1 \times 10^{3}$ conidia $\mathrm{ml}^{-1}$, typical leaf spot symptoms were observed on the leaves of Asalio plants. Disease severity according to the scale was $39.20 \%$. For molecular identification, 650 bp internal transcribed spacer (ITS) regions (ITS1, 5.8s and ITS2) were amplified through polymerase chain reaction (PCR). The nucleotide sequences from ITS regions of the isolates were submitted to NCBI with GenBank accession numbers MA585375.

\subsection{Introduction}

Asalio (Lepidium sativum Linn; Family: Brassicaceae) is a medicinal plant. It is known as "Common cress", "Land cress", "Haliv", "Garden cress" or "Chandrasur" in some regions of India ${ }^{1}$. Asalio in local language is known as "Chandrasur" and considered as an important rabi medicinal crop in India"

Asalio is distributed in various parts of India and Europe including Britain, France, Italy and Germany. Cultivation of Lepidium sativium has been taken up in several parts of India viz., states of Madhya Pradesh, Uttar Pradesh, Rajasthan, Gujarat, Maharashtra etc. In Rajasthan more than 8000 ha area is under its cultivation ${ }^{3}$. According to National Horticulture Board, area of aromatic and medicinal plant in 2017-2018 in India is 650 ha with decrease in annual growth rate $2.11 \%$ as compared to $2016-2017$ and production in India 1037 Million tonnes with increase in growth rate $6.69 \%$ and productivity is 1.6 tonnes/ha in 2017-18.

Lepidium sativum is usually cultivated for its leaves, which are used in salad, sandwiches and seeds with high nutritional value can be exploited as a functional food ingredient ${ }^{4}$. It contains good amount of vitamin $\mathrm{E}$ which is responsible for reducing risks of infertility, inflammation, cardiovascular diseases, neurological disorders, diabetes and certain types of cancers in humans ${ }^{5}$.

It is an annual, erect, glabrous, herbaceous plant growing up to the height of about $20-45 \mathrm{~cm}$. It has raceme inflorescence, white flowers and the pods are round, elliptic, emarginated notched at apex and winged ${ }^{6}$. 
In the year 1967 the Alternaria leaf spot of Asalio was first reported from Kaffa province, Ethiopia and the pathogen was identified as Alternaria brassicae (Berk.) Sacc. ${ }^{7}$

Singh and Upadhyaya, $(1971)^{8}$. Reported first time that Alternaria leaf spot caused by Alternaria alternata on Asalio at College Farm, Banaras Hindu University, Varanasi (U.P.)

Conventional and old identification of phyto-pathogenic fungi was accomplished with colony appearances and morphological characterization; however, these characters show resemblance with many fungal species. The internal transcribed spacer (ITS) regions (ITS1, 5.8s and ITS2) are regarded as standard markers and barcodes for genetic material of many fungal species ${ }^{9}$.

The symptoms produced by Alternaria alternata on Lepidium sativum L. are given in detail by Singh and Upadhyaya, $(1971)^{8}$. The initiation of disease symptom is from basal leaves in the form of small, yellow, circular patches which becomes necrotic, having 2-5 concentric rings and light brown in colour (1-7 mm in diameter). Sometimes these spots coalesce with each other and occupy large blighted area. In later stage, spots become larger in size with distinct concentric rings and dark in colour. The severity of the disease increases upto mid January. At this stage, the stem and floral parts of plant also become diseased. Finally, whole plant shows typical blight symptoms. Fungal colonizes the in xylem of the host plant, and as a result, blockage and breakdown of the xylem lead to wilt disease symptoms such as, leaf wilting, yellowing and eventually the death of the plant.

\subsection{Materials And Methods}

\section{Collection of disease samples :}

Asalio is cultivated medicinal crop. Fristly, I took permission through head of plant pathology department of Rajasthan College of Agriculture, Udaipur from Asalio's farmers to enter selected their farm around the Udaipur district in Rabi season of 2017. Asalio Alternaria leaf spot disease samples were collected from severely Alternaria leaf spot infected farmer's field during survey of department during disease inspection. These farmers were growing local land races of Asalio on their fields as a cultivated crop. The fungal culture of pathogen recovered from these samples was used in present study.

\section{Isolation, purification and pathogenicity tests of the pathogen.}

Fungal pathogen was isolated from collected diseased samples of Asalio using standard methodology on potato dextrose agar (PDA) medium. Small bits of infected portions were surface sterilized for 1 minute in mercuric chloride solution $(0.1 \%)$ and washed thrice in sterilized distilled water under totally aseptic conditions in a laminar air flow. These were then dried by keeping in two folds of sterilized filter papers then aseptically transferred to PDA in Petri plates. The plates were incubated at $27 \pm 1^{\circ} \mathrm{C}$ for $7-8$ days and identified by ITCC (New Delhi) as a Alternaria alternata culture which caused Alternaria leaf spot disease in Asalio and the culture morphological characters were same as previously reported by Utikar and Padule $(1980)^{10}$. He reported that conidiophores of $A$. alternata were simple, light brown, 
variable in length ranging from 17.10 to $61.56 \mu \mathrm{m}$ and mostly 2-3 septate rarely $4-5$ septate. Conidia were found light to dark brown in colour, uniform with 0-2 longitudinal septa and 1-6 transverse septa, and variable in shape and size, mostly oval shape with rudimentary beak and in size measureing about 10.26$77.52 \times 4.56-14.82 \mu \mathrm{m}$. Simmons and Roberts (1993) ${ }^{11}$ observed three-dimensional sporulation patterns of $A$. alternata in electron microscope at 50 magnification.

For proved pathogenicity and fulfilling the Koch's postulates an inoculum with load of $1 \times 10^{3}$ conidia $\mathrm{ml}^{-}$

${ }^{1}$ concentration of the spores was inoculted in pot grown Asalio. The typical concentric ring symptoms appeared within 7-10 days after inoculation and the symptoms are same as previously reported by Melkania (1980) ${ }^{12}$ that Alternaria alternata caused Alternaria leaf spot on leaves of cress at Almora (H.P.) in India.

The fungal culture of pathogen re covered from these samples was used in present study. The pot experiments were carried out at cage house of Plant Pathology, Rajasthan College of Agriculture, Maharana Pratap University of Agriculture and Technology, Udaipur, the study on "Management of Alternaria leaf spot of Asalio Caused by Alternaria alternata." was undertaken during Rabi 2018-2019. Present studies on the aspects viz., symptomatology, isolation, pathogenicity test, effect of age of the host and effect of inoculum density on disease development on pot grown Asalio plants

\section{Effect of plant age on disease development:}

A pot experiment was laid out with five replications of each treatment following completely randomized design (CRD). A Soil mixture containing soil from fields of RCA, Udaipur and FYM (3:1) was used to fill 30 $\mathrm{cm}$ earthen pots. Seeds of susceptible local landrace of Asalio were obtained from Agronomy farm of RCA, Udaipur. These seeds were sown in these pots. Asalio plants of different ages were achieved by staggered sowing at 10 days interval so as to obtain $10,20,30$, and, 40 days old plants for simultaneous inoculation at one time. Five pots for each plant age group (having 10 plants each) were maintained in cage house of Department of Plant Pathology, RCA, Udaipur. The conidial suspension $\left(1 \times 10^{3} \mathrm{conidia} \mathrm{ml}^{-1}\right)$ of pathogen was used for inoculation of different age groups of plants. Inoculation was made by spray inoculation technique using a hand held atomizer. The inoculated plants were kept in humid chamber for $24 \mathrm{hrs}$ and also these were periodically sprayed with distilled water and covered by polythene bags having 2-3 pores and then transferred to cage house and high humidity was maintained throughout the disease development period by frequent irrigations. Observations for disease severity of Alternaria leaf spot were recorded when plants reach the physiological maturity using 0 to 5 scales given by Gawande and Patil (2003).( (Table 1)

Table No. 1: Standard disease rating scale (0-5 scale) Gawande and Patil (2003) for accessing PDI of Alternaria leaf spot of Asalio 


\section{Scale Description of the symptom}
$0 . \quad$ No infection.
1. $\quad 0.1-10.0 \%$ Leaf area infection.
2. $10.1-25 \%$ Leaf area infection.
3. $\quad 25.1-50 \%$ Leaf area infection.
4. $\quad 50.1-75 \%$ Leaf area infection.
5. $\quad 75.1-100 \%$ Leaf area infection.

The per cent disease index (PDI) was calculated using the formula of the McKinney (1923)

Sum of all individual disease rating

Per cent disease index $(\mathrm{PDI})=$ $\times 100$

Total No. of plants assessed $\times$ maximum rating

\section{Effect of inoculum concentration on disease severity :}

A pot experiment was laid out with five replications of each treatment following completely randomized design (CRD). A Soil mixture containing soil from fields of RCA, Udaipur and FYM (3:1) was used to fill 30 $\mathrm{cm}$ earthen pots. Seeds of susceptible local landrace of Asalio were obtained from Agronomy farm of RCA, Udaipur. These seeds were sown in these pots. Five pots for each plant age group (having 10 plants each) were maintained in cage house of Department of Plant Pathology, RCA, Udaipur. The serial dilution technique was uses with simultaneously adjusting four different inoculum densities viz., $1 \times 10^{1}, 1 \times 10^{2}$, $1 \times 10^{3}, 1 \times 10^{4}$ conidia $\mathrm{ml}^{-1}$ using a haemocytometer. The spray inoculation technique was used for inoculations on pot grown plants different concentrations of conidial suspension. The inoculated plants were kept in humid chamber for $24 \mathrm{hrs}$ and also these were periodically sprayed with distilled water and covered by polythene bags having 2-3 pores and then transferred to cage house and high humidity was maintained throughout the disease development period by frequent irrigations. The PDI was calculated by using the formula given below:

\section{Sum of all individual disease rating}

Per cent disease index $(\mathrm{PDI})=$ $\times 100$

Total No. of plants assessed $\times$ maximum rating

Genomic DNA extraction and PCR amplification:- 
The genomic DNA was extracted from representative isolate of $A$. alternata with standard protocol of PrepMan ${ }^{\circledR}$ Ultra sample preparation reagent ${ }^{13}$. The reagents containing DNA fragments of isolate (100 $\mathrm{ML}$ ) was shifted to new sterile $1.5 \mathrm{~mL}$ Eppendorf tubes for purification. The fungal colonies $(3 \mathrm{~mm}$ diameter) was picked from the pure culture and suspended into reagents. The mixture was incubated for 10 minutes at $100^{\circ} \mathrm{C}$ and centrifuged for 2 minutes at $13000 \mathrm{rpm}$. A $50 \mu \mathrm{L}$ supernatant was shifted to new tubes and DNA was stored at $-20^{\circ} \mathrm{C}^{14}$. The ITS from morphologically confirmed $A$. alternata isolate was amplified through PCR with the help of forward and reverse primers ${ }^{15}$. The PCR reaction was carried out according to standard protocol ${ }^{16}$. The genomic DNA was replaced with sterile distilled water in the negative control.

All methods were carried out according to Indian Council of Agricultural Research (ICAR) guidelines and legislation.

\subsection{Results}

\section{Effect of plant age on disease development :}

The susceptible local landrace of Asalio collected from Agronomy farm of RCA was grown in pots for study of effect of plant age on development of Alternaria leaf spot of Asalio following a completely randomized block design with five replications. The sowing dates were adjusted so as to obtain 10, 20, 30 and 40 days old plants in cage house for simultaneous inoculations at one time. Spray inoculation technique was used for inoculation. These Four different stages plants were inoculated by spraying $A$. alternata suspension simultaneously at one time. The inoculum density was adjusted to $1 \times 10^{3} \mathrm{conidia}^{-} \mathrm{ml}^{-}$ 1. A standard 0-5 disease rating scale was used to record the disease severity. The observations were recorded after 10-days of inoculation and per cent disease index (PDI) was calculated.

Age of the host is important aspect influencing the disease development. The experiment was conducted to find out the most susceptible stage of Asalio for maximum disease development (Table 2 and Fig 2 and Fig 3). The data presented in Table.2 show disease incidence at different host age i.e., 10, 20,30 and 40 days old plants when inoculated with $A$. alternata suspension simultaneously at one time. The susceptibility of Asalio plants against Alternaria leaf spot increased with increase in plant age. The minimum per cent disease index was observed on 10 DAS plants with PDI 15.20, followed by 20 DAS with 22.40 PDI then 30 DAS with $40.80 \mathrm{PDI}$ and maximum PDI was observed in 40 DAS plants with 55.60. There was a significant difference observed between PDI expressed among different age of host plants. The results revealed that the maximum susceptibile age of Asalio is 40 DAS and minimum 10 DAS against Alternaria leaf spot disease pathogen.

\section{Table No.2: Effect of host age in relation to Alternaria leaf spot severity under artificially inoculated condition on pot grown Asalio plants}




\begin{tabular}{|c|c|c|}
\hline S. No. & Plant age (DAS) & $\begin{array}{l}\text { Per cent disease index } \\
\left(\left.\mathrm{PD}\right|^{*}\right)\end{array}$ \\
\hline \multirow[t]{2}{*}{1.} & 10 & 15.20 \\
\hline & & $(22.93)$ \\
\hline \multirow[t]{2}{*}{2.} & 20 & 22.40 \\
\hline & & $(28.22)$ \\
\hline \multirow[t]{2}{*}{3.} & 30 & 40.80 \\
\hline & & (39.68) \\
\hline \multirow[t]{2}{*}{4.} & 40 & 55.60 \\
\hline & & $(48.20)$ \\
\hline SEm \pm & & 0.775 \\
\hline C.D. $5 \%$ & & 2.342 \\
\hline C.V.\% & & 5.170 \\
\hline
\end{tabular}

* Mean of five replications

DAS: Days after Sowing

Figures in parentheses are arcsine $\sqrt{ }$ per cent angular transformed values.

\section{Effect of inoculum density of Alternaria alternata on disease development :}

A pot experiment was laid out with five replications of each treatment following completely randomized design (CRD). A Soil mixture containing soil from fields of RCA and FYM (3:1) was used to fill $30 \mathrm{~cm}$ earthen pots. Seeds of susceptible local landrace of Asalio were obtained from Agronomy farm of RCA, Udaipur. These seeds were sown in these pots. Five pots for each plant age group (having 10 plants each) were maintained in cage house. Inoculum with four inoculum densities $\left(1 \times 10^{1}, 1 \times 10^{2}, 1 \times\right.$

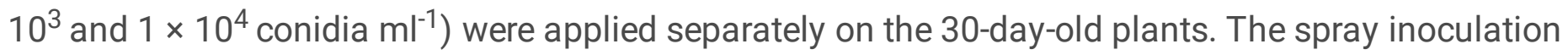
technique was used for inoculations on pot grown plants different concentrations of conidial suspension. Observations for disease severity of Alternaria leaf spot were recorded and calculated PDI when plants reach the physiological maturity.

Among the different tested inoculum concentration levels, it was observed that concentration levels of $1 \times$ $10^{3}$ and $1 \times 10^{4}$ conidia $\mathrm{ml}^{-1}$ exhibited maximum PDI 39.20 and 40.80 respectively. Although, these two concentrations were statistically at par to each other, but also they were significantly superior over concentrations with $1 \times 10^{1}$ and $1 \times 10^{2}$ conidia ml $^{-1}$. The two lower concentrations $1 \times 10^{1}$ and $1 \times$ $10^{2}$ conidia $\mathrm{ml}^{-1}$ exhibited significant difference in the PDI among each other. $1 \times 10^{1}$ was found to 
generate significantly lower PDI (20.00) as compared to $1 \times 10^{2}$ conidia $\mathrm{ml}^{-1}$ with PDI 31.20 ( Table 3, Fig 4 and Fig. 5).

Table No.3: Effect of inoculum density on severity of Alternaria leaf spot of Asalio under artificially inoculated condition on pot grown Asalio plants.

\begin{tabular}{|lll|}
\hline S.No. & $\begin{array}{l}\text { Inoculum density } \\
\left(\text { Conidia } \mathbf{~ m}^{-1} \text { ) }\right.\end{array}$ & $\begin{array}{l}\text { Per cent disease index } \\
(\mathrm{PDI} \text { ) }\end{array}$ \\
\hline 1 & $1 \times 10^{1}$ & $\begin{array}{l}20.00 \\
(26.54)\end{array}$ \\
& & 31.20 \\
2 & $1 \times 10^{2}$ & $(33.93)$ \\
& & 39.20 \\
\hline 3 & $1 \times 10^{3}$ & $(38.74)$ \\
& & 40.80 \\
\hline 4 & $1 \times 10^{4}$ & $(39.68)$ \\
& & 0.938 \\
\hline SEm \pm & & 2.837 \\
\hline C.D. $5 \%$ & & 6.395 \\
\hline C.V.\% & & \\
\hline
\end{tabular}

* Mean of five replications

Figures in parentheses are arcsine $\sqrt{ }$ per cent angular transformed value

\section{Identification of Alternaria alternata:}

The disease severity of alternaria leaf spot in Asalio was $39.20 \%$. Yellow to light-brown small necrotic lesions were recorded during early stage of infection, which turned to dark brown at later stages (Fig 1). The conidiophores of $A$. alternata were simple, light brown, variable in length ranging from 17.10 to 61.56 $\mu \mathrm{m}$ and mostly 2-3 septate rarely 4-5 septate. Conidia were found light to dark brown in colour, uniform with 0-2 longitudinal septa and 1-6 transverse septa, and variable in shape and size, mostly oval shape with rudimentary beak and in size measureing about 10.26-77.52 x 4.56-14.82 $\mu \mathrm{m}$. A novel disease rating scale showing the degree of symptoms was developed for the disease (Fig 1). The isolated culture of $A$. alternata was confirmed with the help of microscope based on morphological properties of conidiophores. After confirmation, a pathogenicity test was carried out to reconfirm the authenticity of this pathogen. The Koch's postulates were applied to confirm pathogenicity on the original host with the help of syringe. After inoculation of pathogen, same type of symptoms were observed after few weeks. 


\section{PCR amplification and identification of nucleotide sequences of pathogen:}

About 650 bp ITS regions from the isolate of Asalio sample were amplified through PCR and GenBank accession numbers were MW585375. The nucleotide sequences from ITS regions of the isolates were submitted to NCBI with GenBank accession numbers MA585375.

\subsection{Discussion}

Since there has been no concrete information till date on relationship between host age on disease development of Asalio Alternaria leaf spot. However, some reviews on this aspect are available on other hosts of the pathogen. Saad and Hagedorn, (1969) ${ }^{17}$ observed that the proneness to susceptibility to attack of $A$. alternata increased with increase the age of host plant. Mishra et. al. (2002) ${ }^{18}$ reported that 8 week old sunflower plants had maximum susceptibility to infection of $A$. alternata. Radish plants were more vulnerable at mature stage as compare to young stage to infection by $A$. raphani observed Sangwan et. al. (2002) $)^{19}$. The results in present investigation are in tune with the afore said reported review. In the present study attempts to find the most susceptible stage of plants of on test host Asalio revealed variations in the per cent disease index (PDI) at different growth stages. 10 days-old plants were found to be immune to disease on artificial inoculations. However, 20 and 30 days old plants developed moderate disease. Maximum disease (55.60 PDI) was observed when inoculations were made on 40days-old plants ( Fig.6 ). What physiological changes occur at this time in relation to host proneness or resistance is not known, but these need to be studied to understand the host pathogen interactions.

There is no information about effect of inoculum density on development of Alternaria leaf spot disease in Asalio is available in literature. Although review envisages that work on inoculum density have been done on other crops. Huguelet \& Kiesling $(1973)^{20}$ observed that 1000 and 5000 spores of $A$. alternata and Helminthosporium sativum respectively, are optimum for producing blight symptoms in Triticum vulgare (wheat). The results in present investigation are in tune with the afore said reported review. Different disease levels were generated through varied inoculum density $\left(1 \times 10^{1}, 1 \times 10^{2}, 1 \times 10^{3}\right.$

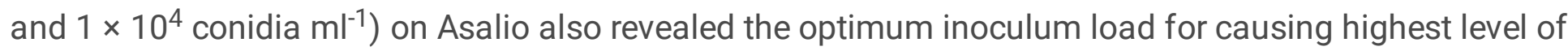
disease at a time. It was found that concentration levels of $1 \times 10^{1}, 1 \times 10^{2}$ conidia ml-1 ${ }^{-1}$ expressed significant differences in PDI (20.00 and 31.20 respectively) in all tested inoculum concentrations. However, inoculum levels of $1 \times 10^{3}$ and $1 \times 10^{4}$ conidia $\mathrm{ml}^{-1}$ were statistically at par to each other with PDI (39.20 and 40.80 respectively). So it is concluded that $1 \times 10^{3}$ conidia $\mathrm{ml}^{-1}$ inoculum concentration was optimum for generating the desired disease (Fig. 7). It suggests that with higher available inoculum concentration A. alternata can cause a good quantum of Alternaria leaf spot on Asalio. It is a limiting factor in realization of good yields.

The morphological characterizations of Alternaria species are unclear because morphological characters of several other fungal species overlap. New molecular tools such as PCR amplification, make reliable identification of fungal pathogen ${ }^{21}$. To overcome this problem, $650 \mathrm{bp}$ ITS regions from three 
representative isolates were amplified through PCR assays with the help of sense and antisense primers $^{22}$ and the evidences of nucleotide sequences were submitted to NCBI for attaining GenBank accession numbers. The obtained nucleotide evidences were used for the accurate confirmation of $A$. alternata on Asalio.

Statistical analysis. Statistical Analysis Software (SAS software) developed by Indian Statistical Research Institute (ISRI), New Delhi was used for performing statistical analyses of data. The critical differences among treatment groups were determined using the ANOVA test at level of significance $(p=$ 0.05). All the experiments were carried out in five replications in Completely Randomized Design (CRD).

The data analysis for this paper was generated using SAS software. Copyright [2014] SAS Institute Inc. SAS and all other SAS Institute Inc. product or service names are registered trademarks or trademarks of SAS Institute Inc., Cary, NC, USA.

\section{Declarations}

\section{Data availability}

Requests for data and materials should be addressed to Dr. Amit Trivedi (email: amit2_trivedi@yahoo.co.in).

\section{Acknowledgements}

I acknowledge the help rendered for this study by Rashtriya Krishi Vikas Yojna (RKVY) Project funded by Government of Rajasthan and Director Research, Maharana Pratap University of Agriculture and Technology, Udaipur, India.

\section{Author contributions}

Dr. Amit Trivedi designed the study and guided throughout all the experiments. P. K. collected the materials and conducted all the experimental methods. P.K. wrote the manuscript with the help of A.D., N. G. and L. K.. A. T. read and revised it, and all the authors approved the final version of manuscript.

\section{Competing interests}

The authors declare no competing interests.

\section{Additional information}

Correspondence and requests for materials should be addressed to P. K..

\section{References}


1. Gokavi, S. S, Malleshi N. G. and Guo, M. 2004.Chemical composition of garden cress (Lepidium sativum) seeds and it fractions and use of bran as a functional ingredient. Journal of Plant Food and Human Nutrition. 59: 105-111.

2. Tiwari, P. N. and Kulmi, G. S. 2004. Performance of Chandrasur (Lepidium sativum) under different levels of nitrogen and phosphorus. Journal of Medicinal and Aromatic Plant Sciences. 26: 479-481.

3. Paroda, R., Dasgupta, S., Bhag Mal, Ghosh, S. P. and Pareek, S. K. 2014. Expert consultation on promotion of Medicinal and Aromatic plants in the Asia-Pacific Region. Proceedings, Bangkok, Thailand. p 259.

4. Eddouks, M., Maghrani, M., Zeggwagh, N. A. and Michel, J. B. 2005. Study of the hypoglycaemic activity of Lepidium sativum $\mathrm{L}$. aqueous extract in normal and diabetic rats. Journal Ethnopharmacology. 97: 391-395.

5. Kulie, T., Groff, A., Redmer, J., Hounshell, J. and Schrager, S. 2009. Vitamin D: an evidence-based review. Journal of America Board Family Medicine. 22: 698- 706.

6. Kumar, A. D. 2006. Ayurvedic Drug Plants, Daya Books Publisher, New Delhi, India, 97.

7. Stewart, R. B. and Dagnalechew Y. 1967. Index of plant diseases in Ethiopia. Exp, Station Bull, No, 30, College of Agriculture, Alenaya, Ethiopia (Spicies, condiments and medicinal plants in Ethiopia, their taxonomy and agricultural significance, Ed. Jansen, P.C.M., Centre for Agricultural Publishing and Documentation, Wageningen, 1981, PP. 216-224).

8. Singh, R. S. and Upadhyaya, J. 1971. Alternaria leaf spot disease of Lepidium sativum. Indian Phytopathology. 24 : 621-622.

9. Hami A, Rasool RS, Khan NA, Mansoor S, Mir MA, et al. (2021) Morpho-molecular identification and first report of Fusarium equiseti in causing chilli wilt from Kashmir (Northern Himalayas). Scientific Reports 11: 1-14.

10. Utikar, P.G. and Padule, D.N. 1980. A virulent species of Alternaria causing leaf blight of onion. Indian Phytopathology. 33: 335-336.

11. Simmons, E. G. and Roberts, R. G. 1993. Alternaria themes and variations. Mycotaxon. 48: 109-140.

12. Melkania, N. P. 1980. Lepidium sativum Linn - A new host record for Alternaria alternata. (Fr.) Keissler. Current Science. 49 : 27-28.

13. Premalatha K, Kalra A (2013) Molecular phylogenetic identification of endophytic fungi isolated from resinous and healthy wood of Aquilaria malaccensis, a red listed and highly exploited medicinal tree. Fungal Ecology 6: 205-211.

14. Ma G, Bao S, Zhao J, Sui Y, Wu X (2021) Morphological and molecular characterization of Alternaria species causing leaf blight on watermelon in China. Plant Disease 105: 60-70.

15. Aloi F, Riolo M, Sanzani SM, Mincuzzi A, Ippolito A, et al. (2021) Characterization of Alternaria Species Associated with Heart Rot of Pomegranate Fruit. Journal of Fungi 7: 172.

16. Ismaiel GH (2018) Antioxidant, Antimicrobial and Anticancer Activities of Egyptian Conocarpus erectus L. leaves Extracts. Egyptian journal of food sciences 46: 165-175. 
17. Saad, S.Y. and Hagedorn, D. J. 1969. Symptomatology and epidemiology of Alternaria leaf spot of bean, Phaseolus vulgaris. Phytopathology. 59: 1530-1533.

18. Mishra, V., Gupta, T. and Srivastava, K. C. 2002. Progress of Alternaria blight of sunflower in relation to environment and host age. Journal of Phytopathological Research. 15: 179-182.

19. Sangwan, M.S., Mehta, N. and Gandhi, S. K. 2002. Some pathological studies on Alternaria raphani causing leaf and pod blight of radish. Journal of Mycology and Plant Pathology. 32:125-126.

20. Huguelet, I. E. and Kiesling, R. L. 1973. Influence of inoculum composition on the black point disease of durum wheat. Phytopathology. 63 : 1220-1225.

21. Ali FS, Akbar A, Prasongsuk S, Permpornsakul P, Yanwisetpakdee B, et al. (2018) Penicillium imranianum, a new species from the man-made solar saltern of Phetchaburi province, Thailand. Pakistan Journal of Botany 50: 2055-2058.

22. Abbas MF (2019) Prevalence and Characterization of Fungal Pathogens Associated with Loquat (Eriobotrya japonica) in Punjab Pakistan: PMAS-Arid Agriculture University, Rawalpindi.

\section{Figures}
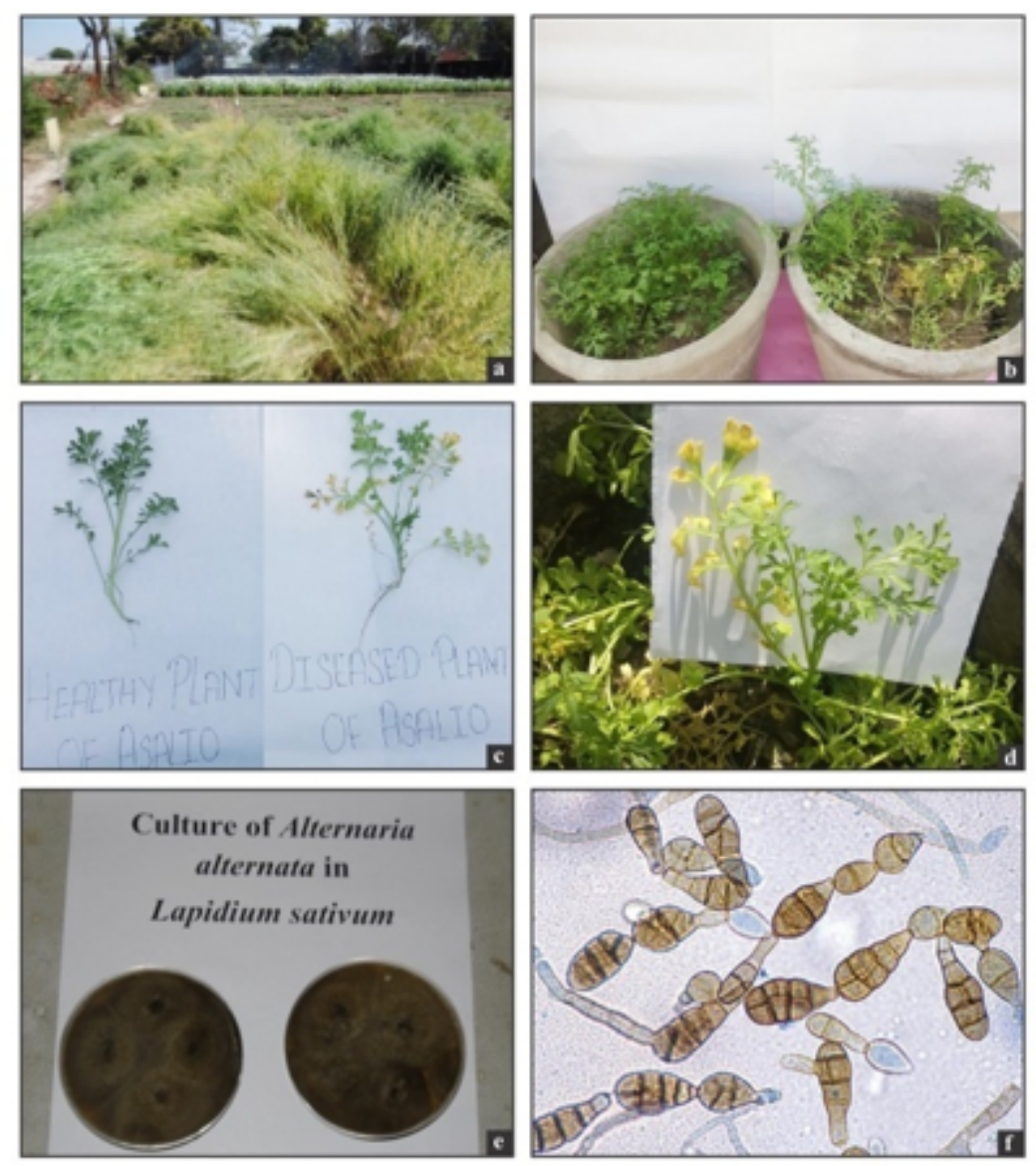

Figure 1 
Pathogenicity of A. alternata on Asalio. a. field view of disease , b. diseased plant c. typical symptom generated by A. alternata, $d$. close view of disease symptoms, e. pure culture of A. alternata and f. conidia at $40 \mathrm{X}$

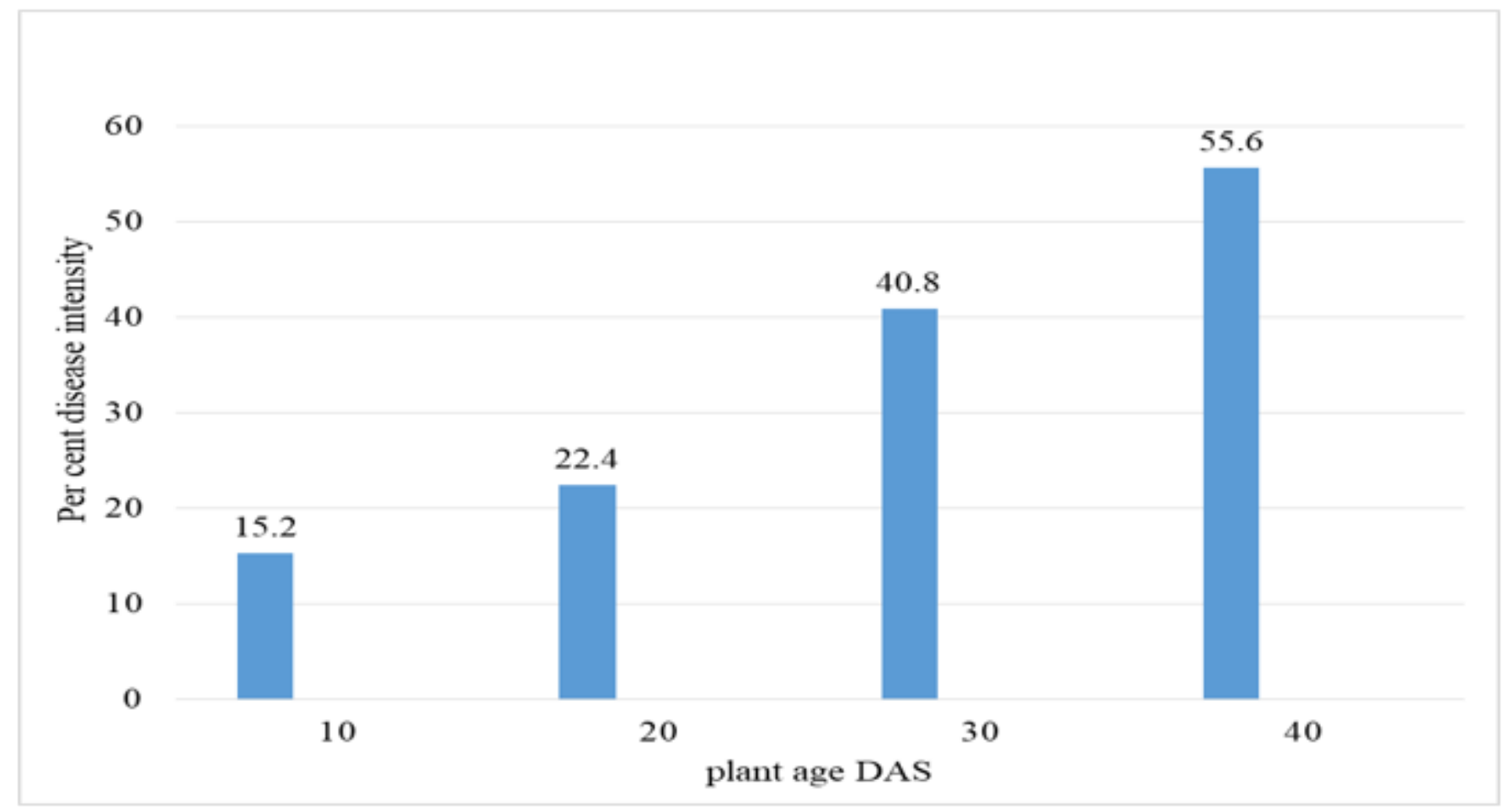

Figure 2

Effect of plant age in relation to Alternaria leaf spot severity under artificially inoculated condition under pot grown Asalio plants. 

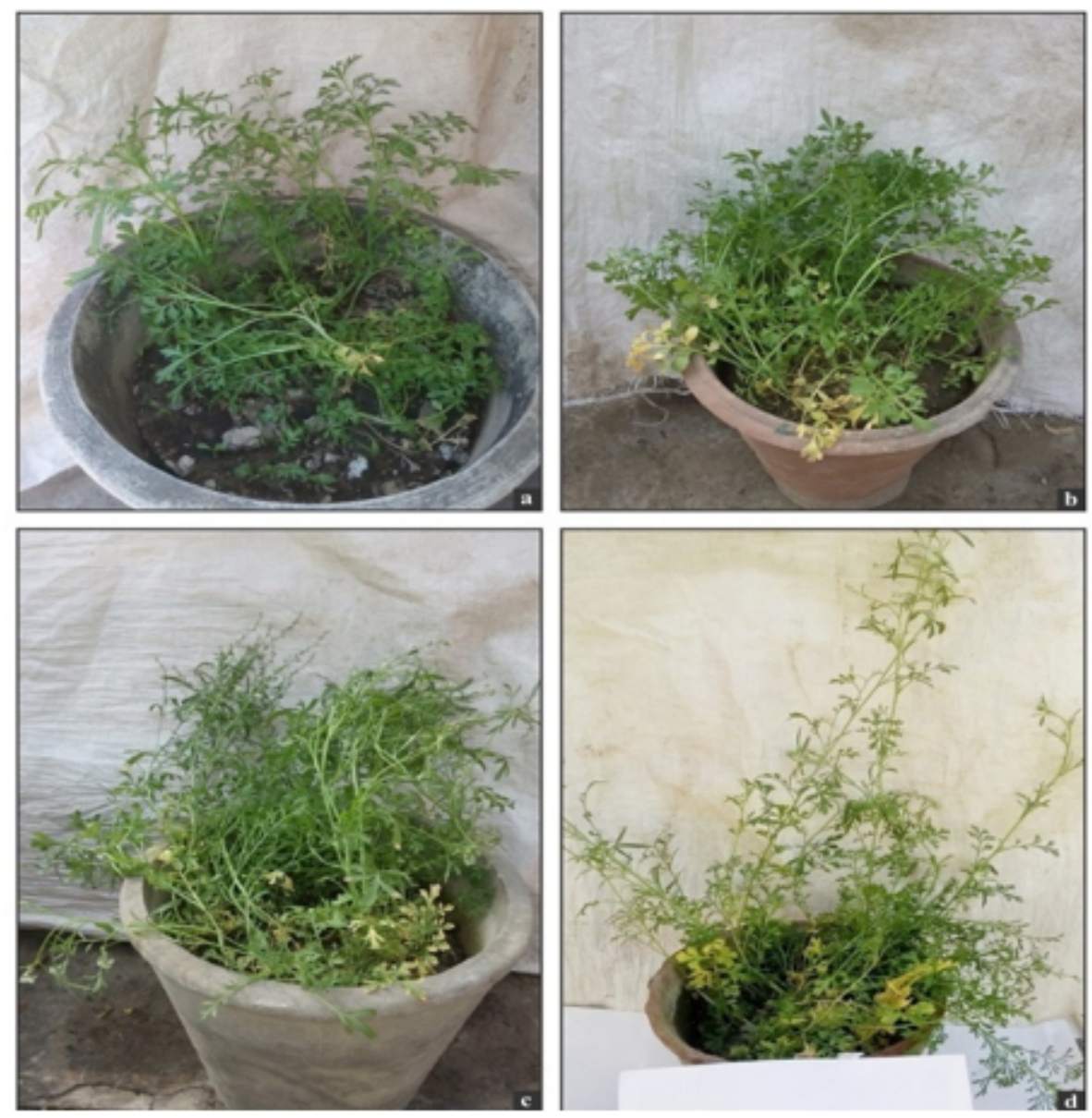

\section{Figure 3}

Effect of plant age in relation to Alternaria leaf spot severity under artificially inoculated condition on pot grown Asalio plants. a. 10 days after sowing , b. 20 days after sowing, c. 30 days after sowing and d. 40 days after sowing. 


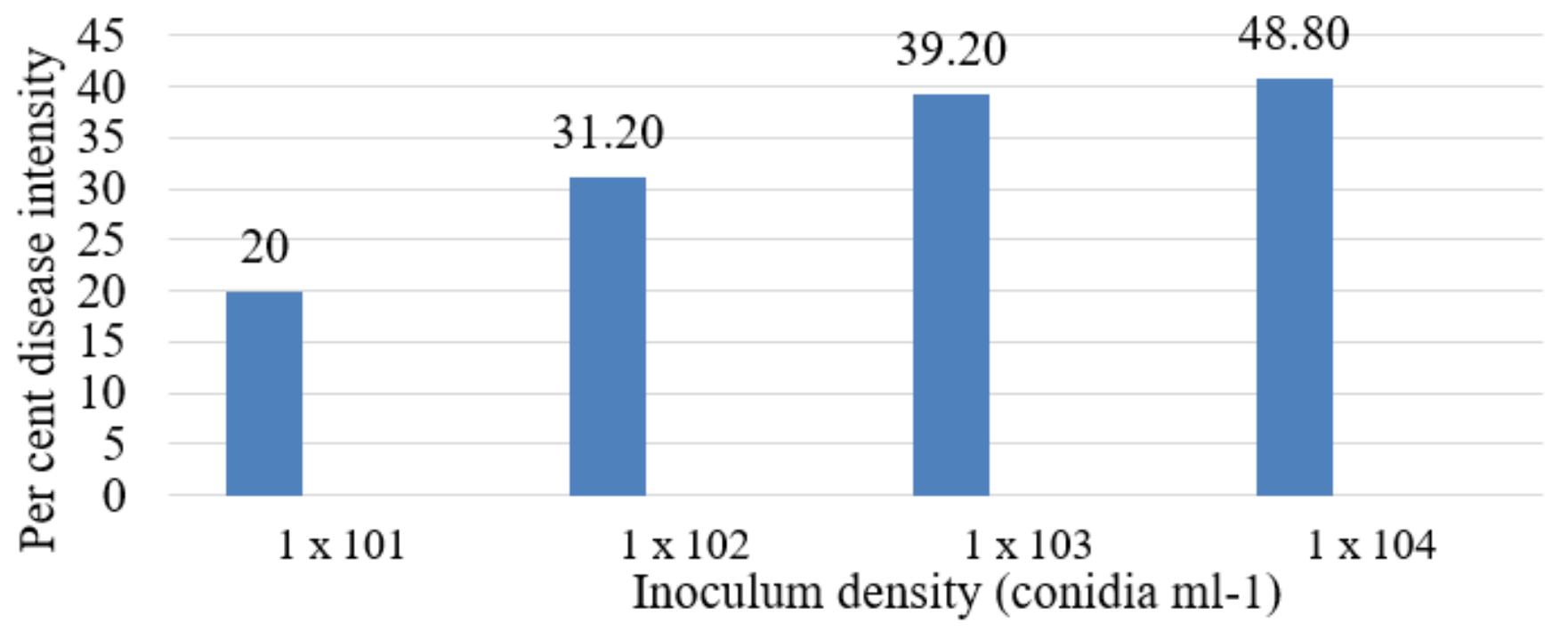

Figure 4

Effect of inoculum density on disease development of Alternaria leaf spot under artificially inoculated pot grown Asalio plants 

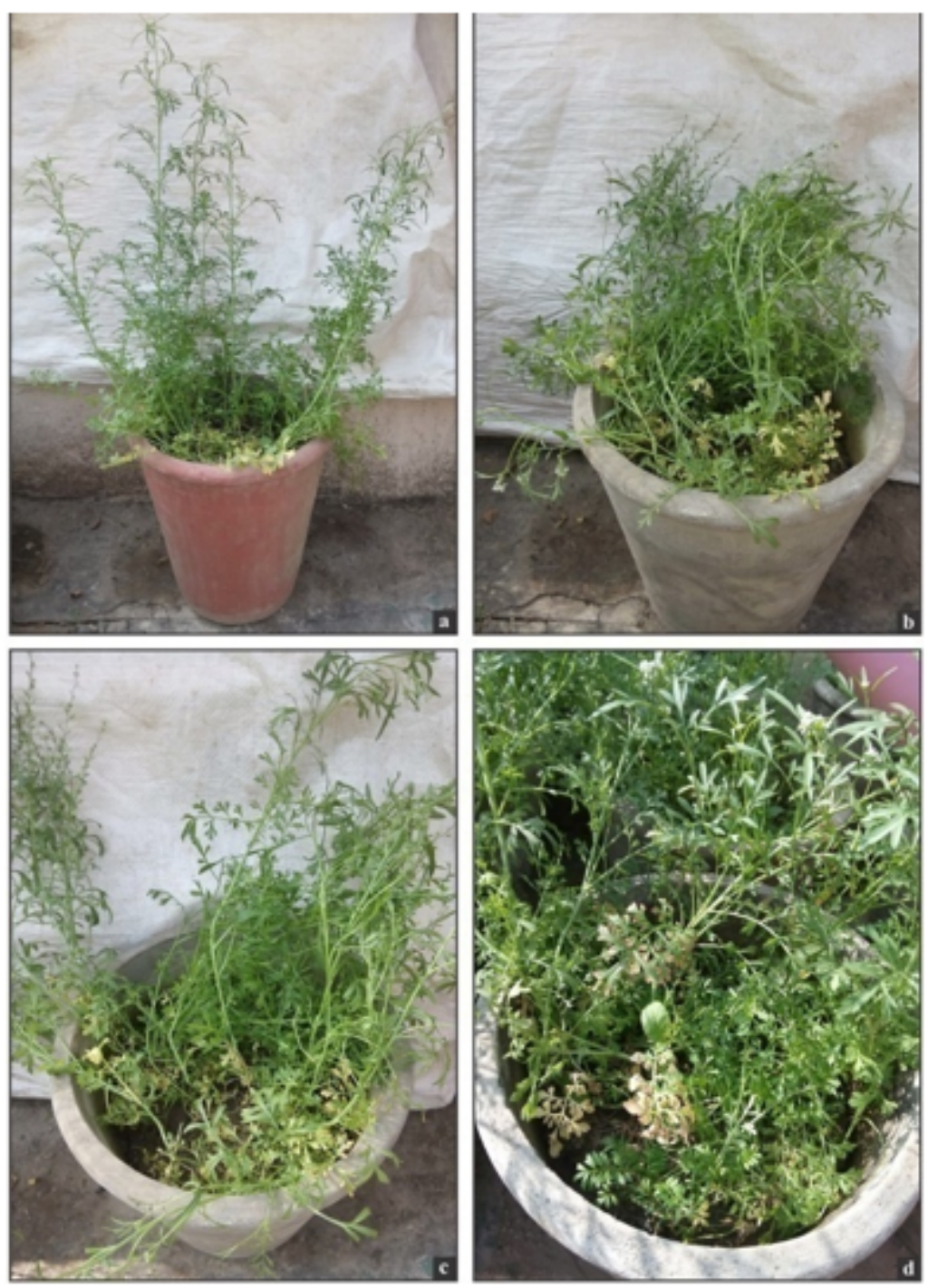

Figure 5

Effect of inoculums density on severity of Alternaria leaf spot of Asalio under artificially inoculated on pot grown Asalio plants. a. 1x 101 conidia ml-1, b. 1x102 conidia ml-1, c. 1x103 conidia ml-1 and d. 1×104 conidia $\mathrm{ml}-1$ 


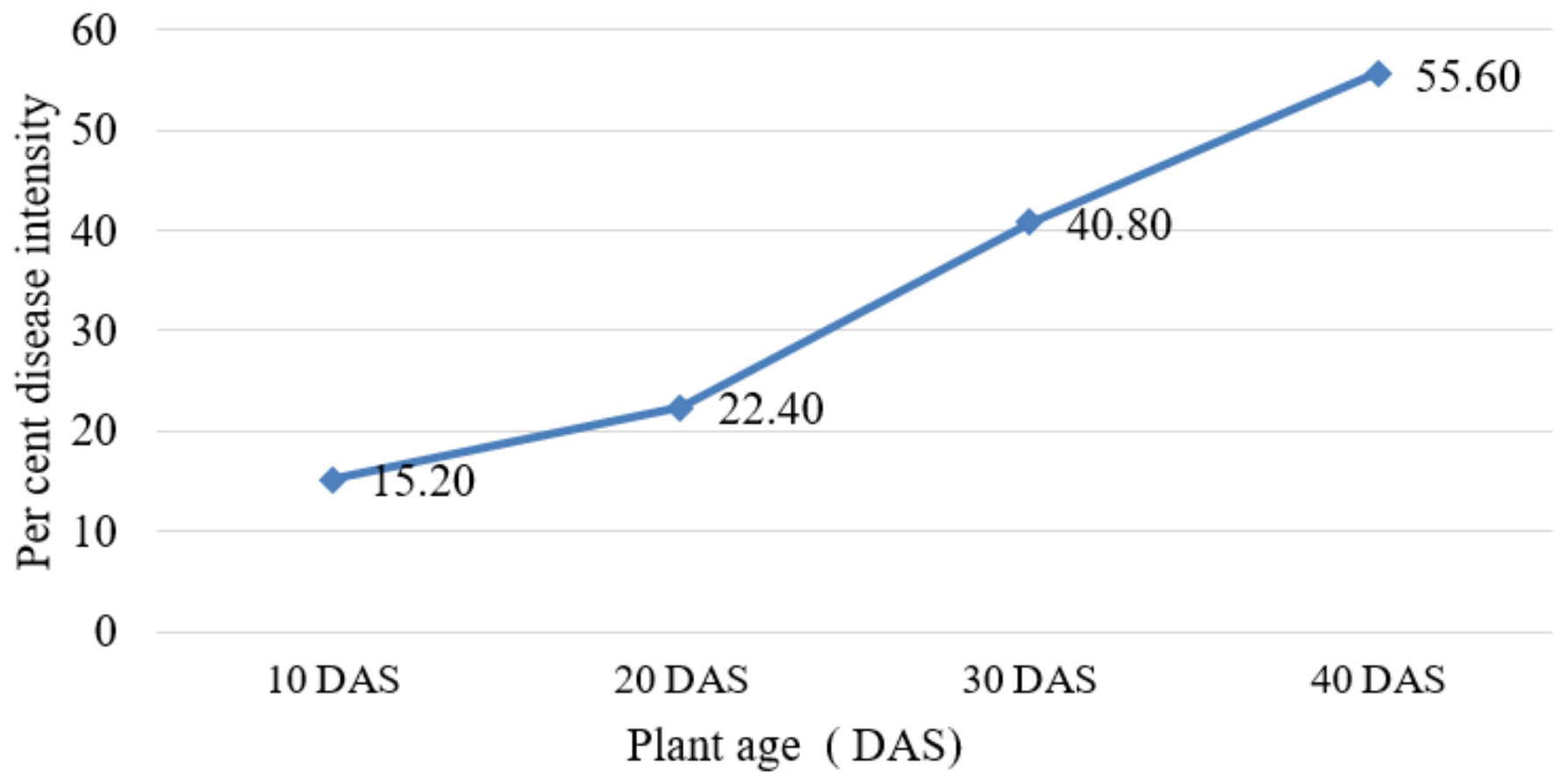

Figure 6

Effect of different host age in relation to Alternaria leaf spot of Asalio 


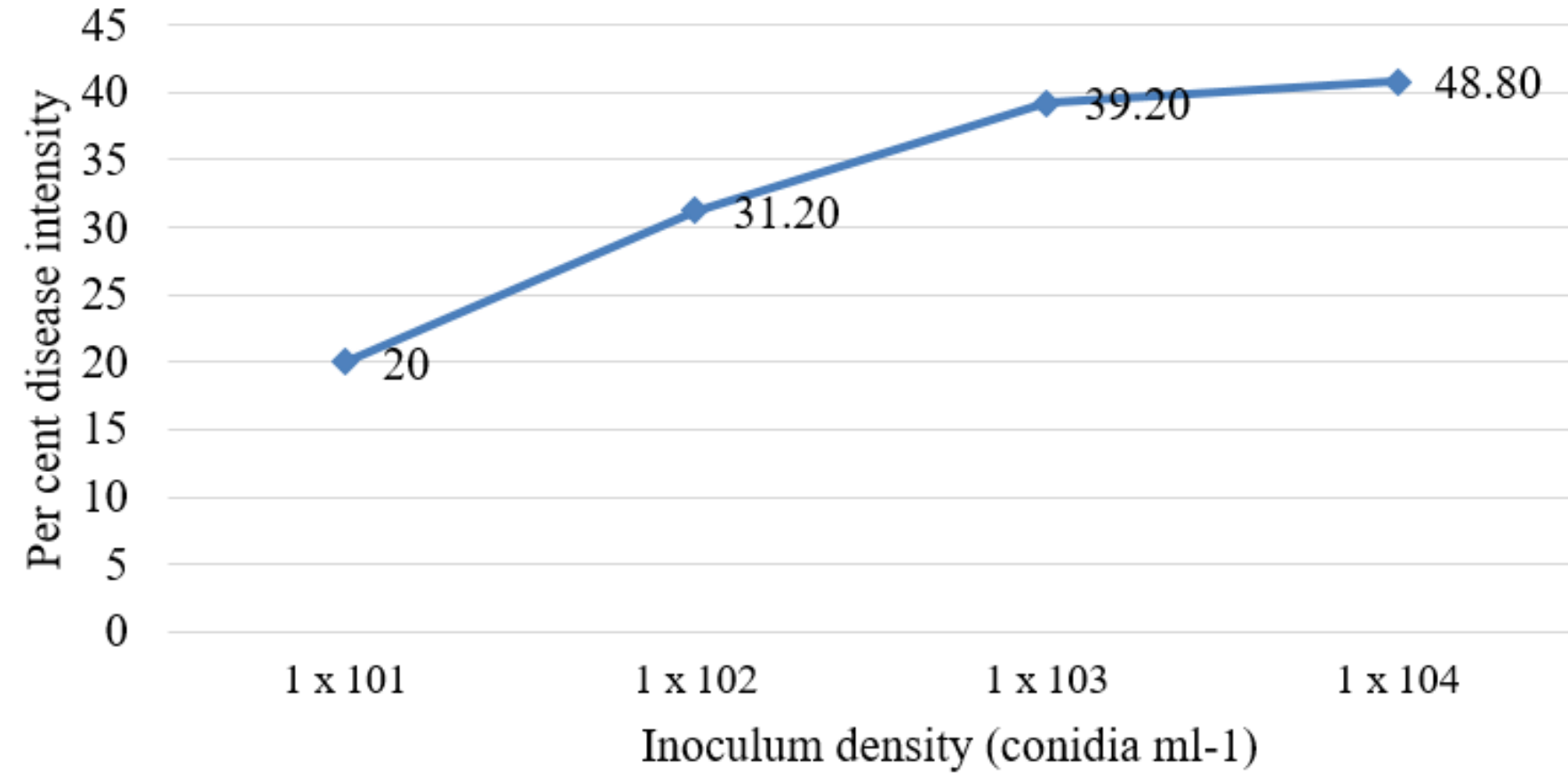

Figure 7

Effect of different inoculum density on disease development of Alternaria leaf spot Asalio. 